\title{
Optimization Analysis of USV Based on Genetic Algorithm
}

\author{
WU Changnan,a , LIU Man ${ }^{1, b}$, YANG Songlin ${ }^{1, c, *}$, GAO Shasha ${ }^{1, d}$ \\ ${ }^{1}$ College of Marine and Shipbuilding Engineering, Jiangsu University of Science and Technology, Zhenjiang, \\ Jiangsu, China \\ a 1570641990@qq.com, b941376267@qq.com, cysl560516@vip.163.com, d1679488425@qq.com \\ *YANG Songlin
}

Keywords: USV (Unmanned Surface Vehicle); Genetic algorithm; Optimization of mathematical model; Comprehensive optimization

\begin{abstract}
USV's hull form optimization is a multi-objective optimization problem, which involves coordination and unification of performance in all aspects. To determine the sensitivity to overall optimization including rapidity, maneuverability, seakeeping and overturning of the USV was an important aspect in the design process.This paper took a new type of unmanned gliding-hydrofoil craft as the research object, by studying the performance of USV, a comprehensive optimization mathematical model of four performance was established. The optimal design variables, objective functions and constraints of the USV were also determined. The optimization program was programmed by genetic algorithm, and the optimization analysis of USV was carried out. In this paper, the performance of each performance objective function was studied with the number of iterations, and different weight schemes were selected to study the influence of four performance on the sensitivity of weight setting. The results showed that when the genetic algebra was 3000 generations, the optimization result was the best; The weight setting had a significant effect on the integrated optimization system, and the rapidity had the greatest impact, the second was maneuverability, and the optimization result could provide some reference value for the preliminary design and optimization analysis of the USV.
\end{abstract}

\section{Introduction}

USV is a small surface tool, because of its small size, flexibility, intelligence, high speed and other characteristics, and can replace the human in the harsh sea conditions to work, it has become an object of widespread concern among scholars both at home and abroad in recent years. Research on all aspects of it was also widespread. With the depletion of land resource consumption, countries have begun to invest a lot of money and manpower to explore the ocean and the development of marine technology. In the military field, because of its flexible use, long battery life, strong functional, can carry equipment and other characteristics, USV can be used as a self-defense and offensive carrier[1-2]. In the civil field, USV has meteorological information monitoring, water quality sample collection, fishery farming, hydrological detection and other functions. In order to make it better to complete the task, the optimization of the performance of USV is very urgent.

At present, some colleges and universities and institute of ship design and research in China, and scientific research institutes abroad carried out relevant research on the performance optimization of USV, and achieved certain results. However, from the existing literature, most of the research was to pursue its unilateral performance, while ignoring the coupling effect of other properties. The traditional ship performance optimization study was to use a simplified method, one or two key outputs as a separate objective function, and other output in the constraint function, in order to solve the optimal value of this single objective function[3-7].Such as: First of all, the optimization of rapidity was studied, and then verify and check the seakeeping and maneuverability etc. But in this way, often at the expense of a performance at the cost of a care for this and lose that result.

This paper took into account the four major performance of USV: rapidity, maneuverability, seakeeping and overturning resistance, and comprehensively optimized the navigation performance 
of USV. Firstly, the basic research and analysis of the genetic optimization algorithm were carried out, and then the comprehensive optimization mathematical model based on the four performance was established. On this basis, the intelligent optimization method was used to write the optimization program, and the optimal calculation of the number of iterations and weights was carried out.

\section{Genetic optimization algorithm}

Genetic algorithm was based on Darwin's natural biological genetic and evolutionary process "natural selection, survival of the fittest" principle and genetic random exchange theory developed a method of optimization[8]. The algorithm had the ability to solve complex system optimization problems.

Genetic algorithm was, by its very nature, a process in which a group was iterated according to evolutionary rules. It began with a possible solution to the problem to be solved, which was also called the initial population. With the initial population, in accordance with the survival of the fittest and the survival of the fittest principle, in each generation, according to the size of each individual fitness to choose a good individual. And then from the selected individuals in accordance with a certain probability of cross and mutation, cross so that the progeny retained the characteristics of the father, the mutation was produced with the parents of different individuals. Thus forming a new population, through the evolution of the generation, to produce more and more excellent population,

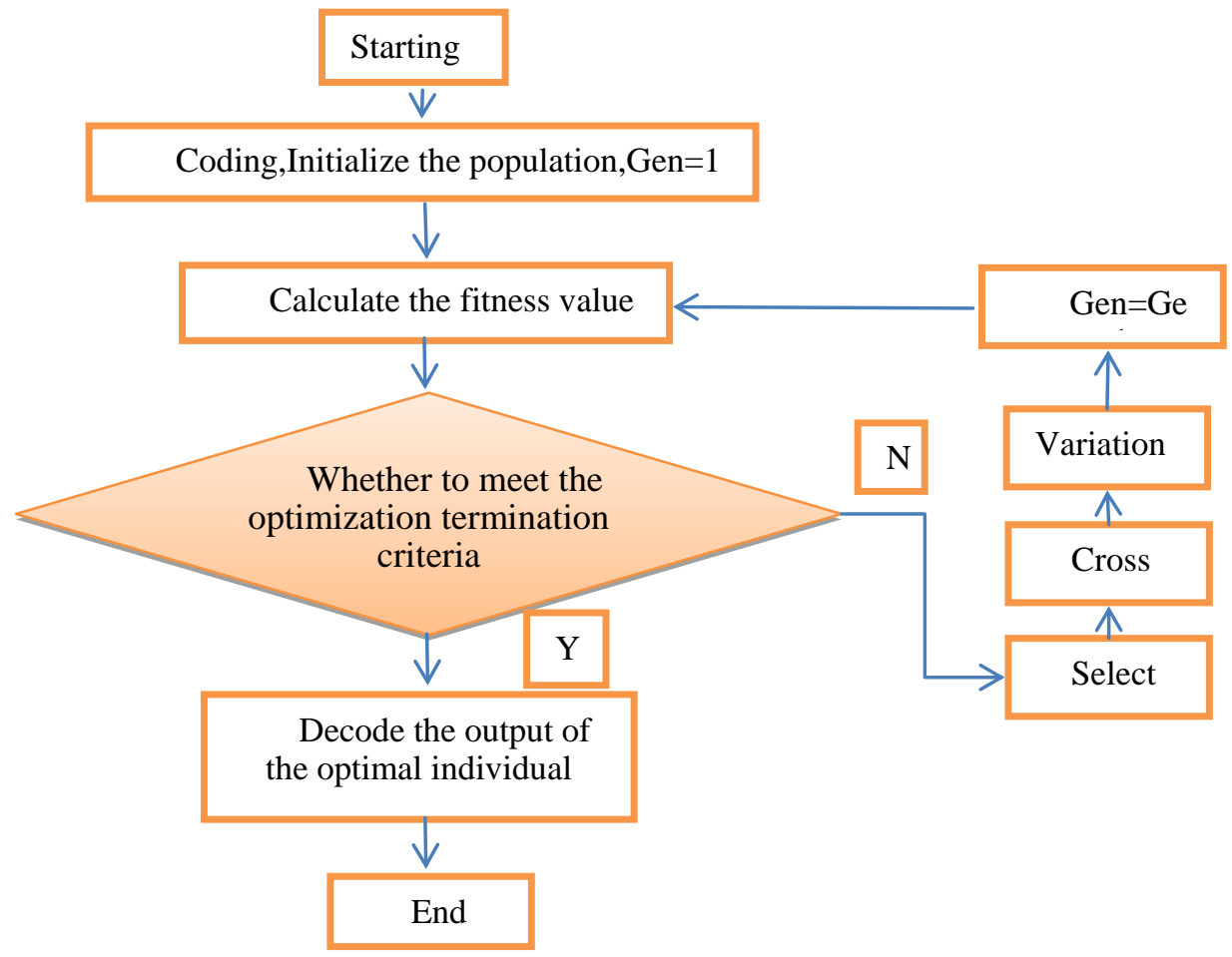

Fig. 1 Genetic algorithm flow chart

that was, the most excellent solution. Genetic algorithm, the population iterative process involved the following aspects: coding methods, the initial population generation, the calculation of fitness, the core of the genetic operation, the termination of these five aspects of the algorithm.The flowchart of the genetic algorithm is show in Fig.1.

\section{Boat type designation}

The USV model in this paper was a new type of USV with splash resistant and hydrofoil. The design effect shown in Fig. 2. The USV's sprinkler and hydrofoil have been calculated by preliminary calculations to determine the basic dimensions of the form. Then, the fine resistance of 
the USV was calculated by fine marine software. The general resistance formula of the USV was fitted, which laid the foundation for the later optimization calculation.

At the same time, in order to facilitate the analysis and calculation of the stability of USV, according to the actual shape of the superstructure of the USV, simplify the figure of the superstructure.

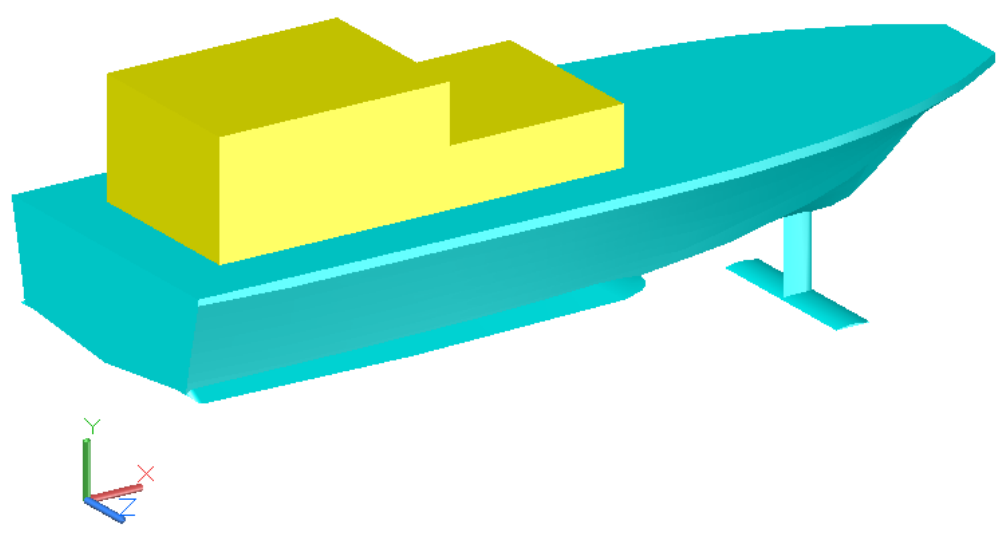

Fig. 2 USV

\section{Comprehensive optimization mathematical model of USV}

The performance of USV was mainly from four aspects: rapidity, maneuverability, seakeeping and overturning. In this paper, the objective function of each performance optimization was established by using the relevant evaluation formula of each performance, and the performance objective function was constructed by means of power exponent product into the performance optimization function of USV. While the floating, thrust, torque, bubble and other aspects of the objective requirements were as a constraint.

\subsection{Design variables}

According to the comprehensive analysis, the length of ship $L$, the ship width $B$, the draft $T$, the square coefficient $C_{B}$, the middle cross section coefficient $C_{M}$, the waterplane surface coefficient $C_{W P}$, the floating heart longitudinal position $x_{b}$, the center of gravity longitudinal position $x_{g}$, the navigation speed $V_{S}$, the sailing angle $\alpha$, propeller speed $N$, propeller disc surface than Aeo, propeller diameter $D P$, pitch ratio $P$ / DP, The ratio of the wingspan to the width of the boat, draft depth ratio $\delta_{T D}$, ramp angle $\beta$, center of gravity from the baseline height of the depth ratio $\delta_{Z D}$, the top floor length and floor length ratio $\delta_{L 1}$, the $\delta_{L B}$ top floor of the superstructure height $\mathrm{H} 1$, the length of the upper floor of the building and the length of the ship $\delta_{L 2}$, the upper floor of the building height $\mathrm{H}_{2}$, Superstructure width and boat width ratio $\delta_{\text {Ba }}$, these 23 parameters were used as design variables of USV.

\subsection{Objective function}

Rapidity, maneuverability, seakeeping, overturning and integrated optimization objective functions were as follows:

(1)Rapidity objective function:

$$
f_{1}(x)=C_{s p}=\frac{V_{S}}{R_{T} / \Delta}\left(\eta_{s} \eta_{R} \eta_{H} \eta_{0}\right)
$$

Where: $R_{T}$ is the hull resistance; $\eta_{S}$ is the shaft system efficiency; $\eta_{R}$ is the relative rotation 
efficiency; $\eta_{H}$ is the hull efficiency; $\eta_{0}$ is propeller open water efficiency.

(2)Maneuvering objective function:

$$
f_{2}(x)=\frac{C^{\prime \alpha_{1}}}{D_{S}^{\alpha_{2}}}
$$

Where: $C^{\prime}$ is dimensionless stability criterion; $D_{S}$ is the minimum relative rotation diameter; $\alpha_{1}$ and $\alpha_{2}$ are their weight.

(3) Seakeeping objective function:

$$
f_{3}(x)=\frac{\mu^{\beta_{1}}}{\left(\psi_{1 / 10}\right)^{\beta_{2}}\left(Z_{1 / 10}\right)^{\beta_{3}}}
$$

Where: $\mu$ is the dimensionless attenuation coefficient; $\psi_{1 / 10}$ is the pitching index; $Z_{1 / 10}$ is the heave indicator;Respectively, $\beta_{1} 、 \beta_{2} 、 \beta_{3}$ are the USV's roll, pitch, dodge the weight of each subsystem.

(4) Anti-overturning objective function:

$$
f_{4}(x)=G M^{\gamma_{1}} * G M_{1}^{\gamma_{2}}
$$

Where: $G M$ is initial stability height; $G M_{1}$ is high stability after overturning; $\gamma_{1}$ and $\gamma_{2}$ are there weights.

Integrated optimization of mathematical models:

$$
f(x)=f_{1}(x)^{\varepsilon_{1}} f_{2}(x)^{\varepsilon_{2}} f_{3}(x)^{\varepsilon_{3}} f_{4}(x)^{\varepsilon_{4}}
$$

Where: $\varepsilon_{1} 、 \varepsilon_{2} 、 \varepsilon_{3} 、 \varepsilon_{4}$ are the weights of rapidity, maneuverability, seakeeping, antioverturning of USV respectively. General requirements them are greater than 0 and $\varepsilon_{1} * \varepsilon_{2} * \varepsilon_{3} * \varepsilon_{4}=1$ are required. The greater the total objective function here, the better the overall performance of the USV.

\subsection{Constraint condition}

(1)Equality constraints

(1) To meet the floating constraints, the optimized drainage volume was basically consistent with the volume of drainage calculated by the given displacement, namely:

$$
\nabla=\operatorname{LBTC}_{B}
$$

(2) Satisfy the thrust constraint: the effective thrust of the propeller was equal to the total resistance of the hull. Which is:

$$
R_{t}=K_{T} \rho n^{2} D^{4}(1-t)
$$

Where: $\rho$ is the water density.

(3) To meet the torque constraint: the torque provided by the host to the propeller was equal to the torque produced by the external force of the propeller. 


$$
K_{Q} \rho n^{2} D^{5}=\frac{P_{s} \eta_{s} \eta_{R}}{2 \pi n}
$$

Where: $P_{S}$ is the host power.

(2) Inequality constraints

(1) To meet the requirements of the bubble: According to Keller formula, the propeller to meet the following inequality conditions:

$$
(1.3+0.3 Z) T_{e} /\left(\left(P_{0}-P_{V}\right) D_{P}^{2}\right)+K-\left(A_{E} / A_{0}\right) \leq 0
$$

Where: $\mathrm{Z}$ is the number of blade number of propeller; $T_{\varepsilon}$ is the thrust force of propeller; $P_{0}$ is the propeller shaft at the center of the static pressure; $P_{V}$ is vaporization pressure of sea water at 15 degrees centigrade; $K$ is a constant, when it is high speed ship, its value is 0 ; when it is oar rigs, its value is 0.1 , when it is single screw ship, its value is 0.2 .

(2) In the condition of the constraint in operation, it was required that the dimensionless check was greater than 0 and the minimum relative rotation diameter was less than 10 .

(3) According to the stability of the ship's specifications, the initial stability was higher than 0.3 , that is: $G M>0.3$.In order to ensure that the USV can self righting after overturning, that is, the ship after overturning is in a state of unstable equilibrium, so $G M_{1}>0$.

\section{Optimization calculation and analysis}

The integrated optimization analysis problem of the USV involves 23 design variables, the upper and lower bounds were shown in table 1 . There were also 4 equality constraints and 4 inequality constraints. This shows that this is a complex engineering optimization problem. In this paper, the above genetic algorithm and the optimized mathematical model were used to write the optimization program to optimize the calculation.

Table 1 Design variables on the upper and lower limits

\begin{tabular}{cccccc}
\hline $\begin{array}{c}\text { Serial } \\
\text { number }\end{array}$ & Design variable name & Symbol & Unit & $\begin{array}{c}\text { Lower } \\
\text { limit }\end{array}$ & $\begin{array}{c}\text { Upper } \\
\text { limit }\end{array}$ \\
\hline 1 & The length of ship & $\mathrm{L}$ & $\mathrm{m}$ & 5.995 & 6.005 \\
2 & Boat wide & $\mathrm{B}$ & $\mathrm{m}$ & 1.921 & 1.925 \\
3 & Draft & $\mathrm{T}$ & $\mathrm{m}$ & 0.365 & 0.37 \\
4 & Block coefficient & $\mathrm{Cb}$ & $/$ & 0.456 & 0.46 \\
5 & Cross section coefficient & $\mathrm{Cm}$ & $/$ & 0.6 & 0.7 \\
6 & Waterline coefficient & $\mathrm{Cwp}$ & $/$ & 0.88 & 0.96 \\
7 & Longitudinal position of center of & $\mathrm{Xb}$ & $/$ & -3 & -2 \\
8 & buoyancy & Pp & $\mathrm{m}$ & 0.205 & 0.208 \\
9 & Propeller diameter & $A_{e o}$ & $/$ & 0.55 & 1 \\
10 & Propeller pitch ratio & $\mathrm{P} / \mathrm{Dp}$ & $/$ & 0.95 & 1.05 \\
11 & Distance from center of gravity /L & $x_{g}$ & $/$ & 0.05 & 0.08 \\
\hline
\end{tabular}




\begin{tabular}{|c|c|c|c|c|c|}
\hline 12 & $\begin{array}{l}\text { The ratio of breadth of the } \\
\text { hydrofoil to beam }\end{array}$ & $\delta_{L B}$ & l & 0.45 & 0.55 \\
\hline 13 & Propeller speed & $\mathrm{N}$ & $\mathrm{r} / \mathrm{min}$ & 5400 & 6000 \\
\hline 14 & Design speed & Vs & kn & 22.5 & 23.5 \\
\hline 15 & Ratio of draft to shape depth & $\delta_{T D}$ & l & 0.45 & 0.48 \\
\hline 16 & Trim angle at sea & $\alpha$ & $\circ$ & 4 & 7 \\
\hline 17 & Rise angle & $\beta$ & $\mathrm{m}$ & 10 & 30 \\
\hline 18 & $\begin{array}{l}\text { Distance between center of } \\
\text { gravity and base line height / D }\end{array}$ & $\delta_{\mathrm{ZD}}$ & l & 0.58 & 0.68 \\
\hline 19 & $\begin{array}{c}\text { Ratio of superstructure top length } \\
\text { to floor length }\end{array}$ & $\delta_{L 1}$ & l & 0.6 & 1 \\
\hline 20 & $\begin{array}{l}\text { The height of top floor of the } \\
\text { superstructure }\end{array}$ & $\mathrm{H} 1$ & $\mathrm{~m}$ & 0.2 & 0.5 \\
\hline 21 & $\begin{array}{l}\text { Ratio of upper floor building } \\
\text { length to length of the ship }\end{array}$ & $\delta_{L 2}$ & l & 0.6 & 0.8 \\
\hline 22 & $\begin{array}{l}\text { The upper floor of the building } \\
\text { height }\end{array}$ & $\mathrm{H} 2$ & $\mathrm{~m}$ & 0.2 & 0.5 \\
\hline 23 & $\begin{array}{l}\text { Ratio of superstructure width to } \\
\text { boat width }\end{array}$ & $\delta_{\text {Вa }}$ & l & 0.6 & 0.8 \\
\hline
\end{tabular}

All aspects of the weight settings were as follows:

$$
\begin{aligned}
& \varepsilon_{1}=2, \varepsilon_{2}=1.6, \varepsilon_{3}=0.625, \varepsilon_{4}=0.5 ; \alpha_{1}=1.6, \alpha_{2}=0.625 ; \\
& \beta_{1}=0.625, \beta_{2}=1.6, \beta_{3}=1 ; \gamma_{1}=1.6, \gamma_{2}=0.625
\end{aligned}
$$

Different generations of genetic algorithms were optimized for computation on a single computer which highest frequency is $2.8 \mathrm{GHz}$, memory is $3.49 \mathrm{~GB}$, hard disk is 1 TB.

\subsection{Optimal calculation of different genetic generations}

The initial population size of the genetic algorithm was set to 200, the number of inheritance was 1000 to 5000 , the mutation probability was 0.15 , The crossover probability was 0.75 , and we choose growth mechanism strategies. 
Table 2 Genetic algorithm results for different iterations

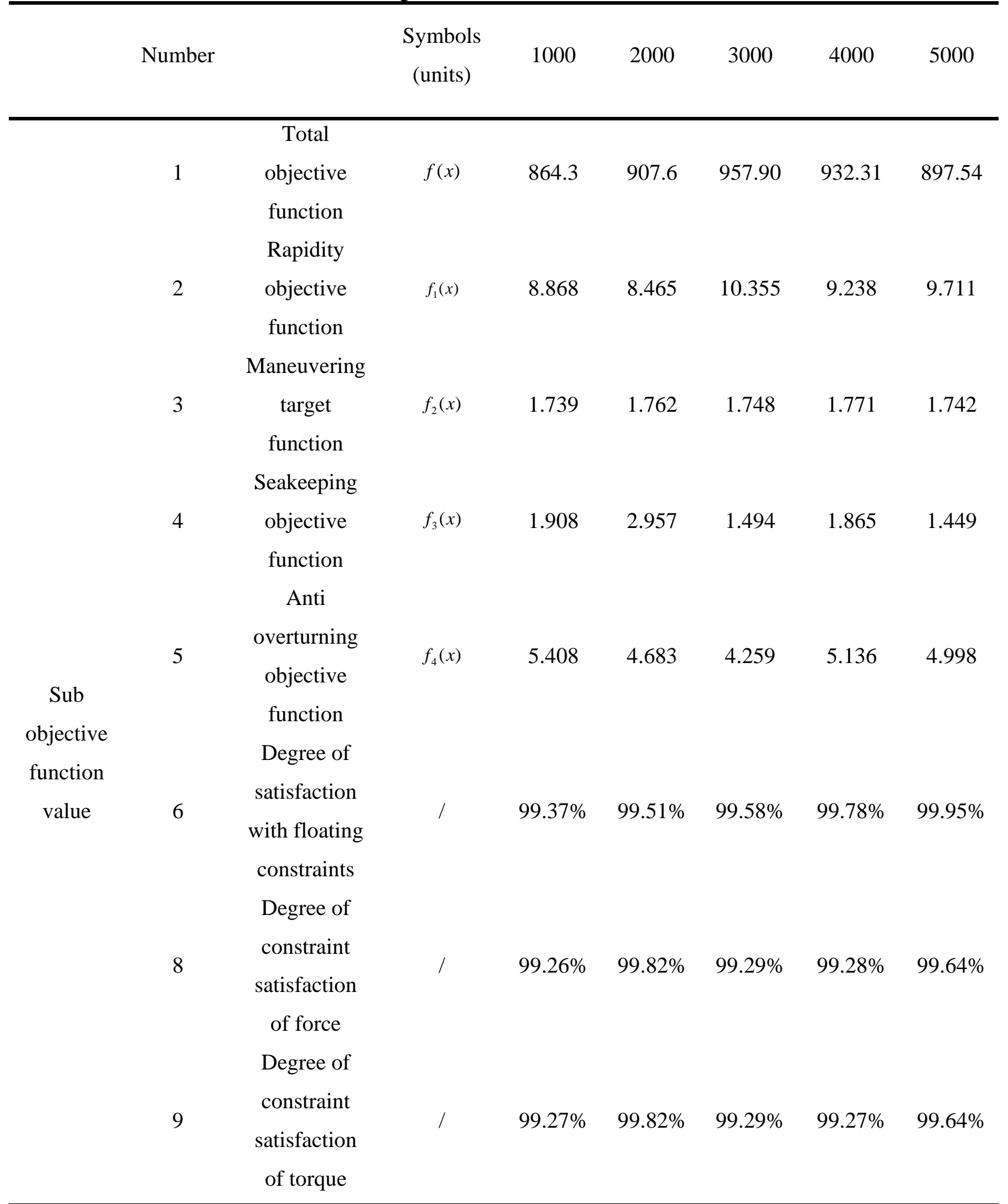

According to the above calculation results, it can be found that the total objective function value fluctuates with the number of iterations. Generally speaking, the value of the objective function increases and becomes stable as the number of iterations increases. The situation here was caused by the termination condition of the genetic algorithm. In the genetic algorithm, we set the maximum iteration number as the termination condition of the algorithm. This method was simple but not accurate. Because there was no guarantee that the algorithm converges exactly at the maximum iteration.

Further analysis shows that the number of iterations was 3000 generations, and the objective 
function value was the largest in the same group. At this point, the rapidity of the objective function value reached a maximum of 10.355 while other performance objective functions were smaller in the same type. It was shown that the total objective function value at this time was mainly determined by the rapidity of reaching the maximum. Then, the variation of the four performance objective function values with the number of iterations can be observed, and they can also be found to have volatility. The maneuverability and seakeeping and overturning the objective function values in these changes also showed the synchronization, which law of its increase and decrease was basically consistent. It can be concluded the maneuverability and seakeeping and anti overturning between these three properties were not contradictory. To improve the maneuverability of the USV will not have much impact on seakeeping and overturning. It can also be seen that the objective function values of other functions decrease when the objective function value of rapidity increases. This indicates that there was inconsistency between rapidity and several other properties, and that improving rapidity will weaken other performance accordingly. Of course, because our genetic algorithms have drawbacks in termination conditions, we may miss the best values for each iteration.

Finally, we analyzed the degree of satisfaction from the constraints and found that the satisfaction of each group was above $99.2 \%$. It shows that the optimization results of each group have reached the requirements in the constraint conditions.

\subsection{The preliminary study of performance weights}

In the above optimization analysis, our weight settings may not be the most appropriate to the genetic algorithm. Theoretically, the weight setting was based on the degree of emphasis on performance in each area, however, the setting of weights was given before no quantitative criteria were given before. It was necessary to choose a weight scheme that can make the performance of every aspect reach a good level in the application of the algorithm.

Taking into account the optimization of the mathematical model involved more weight. Therefore, we only considered the weight of the four performance objective functions in the comprehensive optimization objective function. Here we followed the rules of setting the weights above, Guarantee $\varepsilon_{1}^{*} \varepsilon_{4}=1, \varepsilon_{2}^{*} \varepsilon_{3}=1$, at the same time, the weight of the rapidity objective function was varied by 0.2 and the weight of the maneuverability objective function was varied by 0.1 . The weights of the remaining objective functions can be derived from the above formula. The specific weight scheme was as follows.

(1) Scenario one: $\varepsilon_{1}=1.8, \varepsilon_{2}=1.5, \varepsilon_{3}=0.666666667, \varepsilon_{4}=0.555555556$;

(2) Scenario two: $\varepsilon_{1}=1.8, \quad \varepsilon_{2}=1.6, \varepsilon_{3}=0.625, \varepsilon_{4}=0.555555556$;

(3) Scenario three: $\varepsilon_{1}=1.8, \varepsilon_{2}=1.7, \varepsilon_{3}=0.588235294, \varepsilon_{4}=0.555555556$;

(4) Scenario four: $\varepsilon_{1}=2, \quad \varepsilon_{2}=1.5, \varepsilon_{3}=0.666666667, \varepsilon_{4}=0.5$;

(5)Scenario five: $\varepsilon_{1}=2, \varepsilon_{2}=1.6, \varepsilon_{3}=0.625, \varepsilon_{4}=0.5$;

(6) Scenario six: $\varepsilon_{1}=2, \quad \varepsilon_{2}=1.7, \varepsilon_{3}=0.588235294, \varepsilon_{4}=0.5$;

(7) Scenario seven: $\varepsilon_{1}=2.2, \varepsilon_{2}=1.5, \varepsilon_{3}=0.666666667, \varepsilon_{4}=0.454545455$;

(8) Scenario eight: $\varepsilon_{1}=2.2, \quad \varepsilon_{2}=1.6, \varepsilon_{3}=0.625, \varepsilon_{4}=0.454545455$;

(9) Scenario nine: $\varepsilon_{1}=2.2, \varepsilon_{2}=1.7, \varepsilon_{3}=0.588235294, \varepsilon_{4}=0.454545455$;

The above scheme was carried out around the initial weight setting scheme, which purpose was to select a better weight setting scheme. Through the preceding calculation, the performance of each aspect can be better at the 4000 generation times. So we set the number of inheritance to 4000 to calculate. 
Table 3 Optimization of different weight schemes

\begin{tabular}{ccccccccccc}
\hline Schemes & 1 & 2 & 3 & 4 & 5 & 6 & 7 & 8 & 9 & $\begin{array}{c}\text { Average } \\
\text { value }\end{array}$ \\
\hline $\begin{array}{c}\text { Objective } \\
\text { function value }\end{array}$ & 817.0 & 951.3 & 581.2 & 1212.1 & 1159.9 & 1297 & 806.6 & 1436.6 & 1429.8 & 1076 \\
Rapidity & 10.1 & 11.2 & 10.2 & 10.4 & 9.9 & 11.1 & 6.9 & 10.1 & 10.3 & 10.0 \\
Maneuverability & 1.7 & 1.7 & 1.7 & 1.8 & 1.8 & 1.8 & 1.7 & 1.8 & 1.8 & 1.8 \\
Seakeeping & 2.4 & 2.7 & 0.9 & 2.3 & 2.5 & 1.6 & 2.8 & 1.5 & 1.2 & 2.0 \\
anti-overturning & 5.2 & 4.3 & 4.6 & 4.5 & 4.8 & 5.2 & 5.0 & 4.6 & 4.8 & 4.8 \\
\hline
\end{tabular}

We set the number of inheritance of genetic algorithm at 4000 times to calculate the above nine schemes. In order to facilitate the analysis and comparison, the average values of the performance objective function and the total objective function were obtained as the reference benchmark. Because the different weight schemes have great influence on the total objective function value, without loss of generality, we compared the advantages and disadvantages of each scheme only from the performance target function values. As a result, it was easy to find that none of the nine sets can satisfy the performance objective function values greater than the average. Among them, there were three performance objective function values can be greater than the average value of the program were scheme one, four or five, six, nine. The percentage difference between the performance objective function values and the mean values that are not satisfied in the five sets of programs was as follows:

Table 4 Unsatisfied Performance Target The difference between the value and the mean value as a percentage of the mean

\begin{tabular}{cccccc}
\hline Schemes & 1 & 4 & 5 & 6 & 9 \\
\hline $\begin{array}{c}\text { Performance } \\
\text { objective }\end{array}$ & 1.722 & 4.542 & 9.829 & 1.576 & 1.178 \\
$\begin{array}{c}\text { function value } \\
\text { Average }\end{array}$ & 1.7533 & 4.7783 & 10.011 & 1.9995 & 1.9995 \\
value & 0.0313 & 0.2363 & 0.182 & 0.4235 & 0.8215 \\
$\begin{array}{c}\text { D-value } \\
\text { Percentage }\end{array}$ & 1.78 & 4.94 & 1.82 & 21.18 & 41.08 \\
\hline
\end{tabular}

According to the difference between the percentage of the table, we could draw the program one, five and four weight setting program was better. With these three programs could be a good balance between all aspects of performance. At the same time, the comparison of these three schemes could also be found, the program side was focused on the anti-wave and anti-overturning; the program four focused on the fast, maneuverability; program five focused on maneuverability and wave resistance. When we carried out the optimization analysis of USV, we could choose the corresponding scheme according to the degree of gravity of USV.

\section{Conclusion}

In this paper, the geometry model of USV with hydrofoil and anti splash strip was established according to the morphological characteristics of USV. Then the geometric parameters were selected as design variables according to the geometric model. At the same time, according to the 
formula of evaluating ship performance, a comprehensive optimization mathematical model taking into account the four major performances was established. Through consulting relevant documents and materials, the weights of the mathematical model were preliminary set up. Optimized calculation was carried out by the program written based on optimized mathematical model and genetic algorithm. A preliminary analysis of the results of the calculations was made. At the same time, different performance weighting schemes were also studied and analyzed. The results show that rapidity has the greatest impact on the overall optimization system and the performance index had a significant influence on the total objective function. The performance weights were different and the optimization results were different. In the design of ships, the requirements of the performance indexes were necessarily different because of the actual navigational conditions and the requirements of the designation. Therefore, how to choose the weight of each performance index was becoming more and more important. In this paper, a better weight scheme was given, which could provide reference for further optimization of USV.

\section{Acknowledgement}

This subject was supported by National Natural Science Foundation of China under Grant No. 51379094.

\section{References}

[1] LIU Chenguang, CHU Xiumin, WU Qing, WANG Guichong. A Review and Prospect of USV Research[J]. Shipbuilding of China, 2014, 55(4): 194-205.

[2] LIU Chenguang, CHU Xiumin, XIE Shuo, YAN Xinping. Review and Prospect of Ship Intelligence[J]. SHIP ENGINERING,2016,(03):77-84+92.

[3] Li Xuebin. Multi-objective evolutionary algorithm in the optimal design of the detaileddesign[J]. MARINE TECHNOLOGY,2008,(04):10-12.

[4] Li Xiaodong. Effect on the Maneuverability and the Main Derivatives of Hydrodynamic by the Design Variables of Ship[D]. Wuhan University Of Technology,2014.

[5] DONG Liang, YANG Songlin. Overall Optimization of Rapidity and Maneuverability of Highspeed Monohull Ship Based on Chaos Algorithm[J]. JOURNAL OF JIANGSU UNIVERSITY OF SCIENCE AND TECHNOLOGY, 2007, 21(1).

[6] WEI Zifan, YU Qiang, YANG Songlin. Optimization analysis of unmanned submersible vehicle based on particle swarm optimization[C]. China International Conference on High Performance Marine Vessels(HPMV), April 10, 2014.

[7] WANG Mengyun. Multidisciplinary Design Optimization Method of Maneuverability, Rapidity and Energy System for Flat UUV[D]. Jiangsu University of Science and Technology, 2015.

[8] CHEN Peng, YANG Song-lin, LIU Fu-wei, YU Qiang. The comprehensive optimization of UUV's performance based on parallel genetic chaos method[J]. Ship Science and Technology, 2013, 35(8):66-71.

[9]Xu Q. Multi-objective based course-keeping controller optimization of Unmanned Surface Vehicle[C]// Control Conference. IEEE, 2014:7483-7486.

[10] SONG Lei. Global autonomy: the future development and key technologies of unmanned surface craft abroad[J]. Military Digest, 2015,(13):26-28. 